\title{
Optimization of dispersed carbon nanoparticles synthesis for rapid desulfurization of liquid fuel
}

\author{
Effat Kianpour $^{1} \cdot$ Saeid Azizian ${ }^{1}$
}

Received: 21 December 2014/Published online: 27 November 2015

(c) The Author(s) 2015. This article is published with open access at Springerlink.com

\begin{abstract}
Stringent regulations and environmental concerns make the production of clean fuels with low sulfur content compulsory for the petroleum refining industry. Because of ease of operation without high energy consumption, the adsorption of sulfur compounds seems the most promising process. Central composite design was used to optimize parameters influencing the synthesis of dispersed carbon nanoparticles (CNPs), a new class of sorbents, in order to obtain an excellent adsorbent for desulfurization of liquid fuel. The optimized dispersed CNPs, which are immiscible in liquid fuel, can effectively adsorb different benzothiophenic compounds. Equilibrium adsorption was achieved within 2 min for benzothiophene, dibenzothiophene, and 4,6-dimethyldibenzothiophene with removal efficiency values of $75 \%, 83 \%$, and $52 \%$, respectively. The rate of desulfurization by the prepared CNPs in the present work is seven times higher than the previously reported CNPs. Optimized CNPs were characterized by different techniques. Finally, the effect of the mass of CNPs on the removal efficiency was studied as well.
\end{abstract}

Keywords Carbon nanoparticles - Liquid fuel · Adsorption · Central composite design · Desulfurization

Saeid Azizian

sazizian@basu.ac.ir; sdazizian@yahoo.com

1 Department of Physical Chemistry, Faculty of Chemistry, Bu-Ali Sina University, Hamedan 65167, Iran

Edited by Xiu-Qin Zhu

\section{Introduction}

Sulfur compounds which are naturally present in unrefined oils and transportation fuels severely deactivate most catalysts, such as those used in automotive emissions control, petrochemicals production, and fuel cells (Shi et al. 2011). Furthermore, sulfur-containing fuels produce $\mathrm{SO}_{X}$ during combustion, and this seriously affects the environment. Therefore, stringent regulations are imposed on the presence of sulfur in fuel and have caused a growing demand for ultra-low sulfur fuels ( $<10 \mathrm{ppmw}$ ) (Liu et al. 2012). To produce low sulfur-containing fuels, various processes including hydrodesulfurization (HDS) (Song and Ma 2003; Farag et al. 1998; Lara et al. 2005), oxidation (Jiang et al. 2003; Yang et al. 2007), extraction (Bösmann et al. 2001; Zhang et al. 2004), and adsorption (Blanco-Brieva et al. 2011; Hernandez et al. 2010; Fallah and Azizian 2012a, b; Fallah et al. 2012a, b, 2014; Song et al. 2013) have been proposed. HDS, the major technology in current industries not only operates in difficult and costly conditions but also is inefficient in removing the more refractory sulfur compounds such as benzothiophene (BT), dibenzothiophene (DBT), and 4,6-dimethyldibenzothiophene (DMDBT) (Liu et al. 2012). Because of straightforward operation without high energy consumption, the adsorption of organosulfur compounds is an efficient alternative to the conventional HDS process. Recently, dispersed carbon nanoparticles (CNPs) in an aqueous phase was reported (Fallah and Azizian 2012a; Fallah et al. 2012) as a new class of sorbents in removing of BT, DBT, and DMDBT from liquid fuel, and it was shown that the adsorption of different organosulfur compounds on this adsorbent was rapid and had a good selectivity. The synthesis of CNPs is affected by different parameters (volume of water (W), time of irradiation $(t)$, polyethylene glycol (PEG) volume, and 
weight of glucose $(\mathrm{G})$ ) which may have an effect on adsorption and removal efficiency. The main objective of the present work is to optimize these factors to enhance the desulfurization efficiency of CNPs.

Traditionally, a one-variable-at-a-time methodology, which involves changing one variable while keeping the other factors constant, has been used in assessing the effect of factors on the experimental response (Bezerra et al. 2008). This is time consuming and ignores the interactions among various parameters, so it is not an effective optimization technique (Puri et al. 2002). In order to overcome this problem, multivariable optimization methods should be carried out. Response Surface Methodology (RSM), which includes factorial design and regression analysis, is among the most relevant techniques (Bezerra et al. 2008; Puri et al. 2002). This approach has been recently applied for optimization of the production of microporous-activated carbon from various resources (Asenjo et al. 2011). Before using the RSM methodology, we needed to select an experimental design and to fit an adequate mathematical function for estimating the optimum points. Central composite design (CCD) is a symmetrical second-order experimental design most utilized for the improvement of procedures (Bezerra et al. 2008). In this work, central composite design was selected to study simultaneously the effects of four synthesis variables on the desulfurization efficiency as an experimental response and to get the optimum conditions. Based on this method, we arrived at the conclusion and conditions where desulfurization of liquid fuel can be done easily at the highest rate (within less than $2 \mathrm{~min}$ ).

\section{Experimental}

\subsection{Materials}

+D-Glucose, PEG-200, and heptane (purity >99\%) were provided by Merck Co. (USA). Extra-pure reagents of BT, DBT, and DMDBT (mass fraction purity of $>98 \%$ ) were purchased from Sigma-Aldrich Co. (USA). CNPs were prepared following literature procedure (Zhu et al. 2009) but with changes in the amounts of reactants and period of irradiation time. After dissolving +D-glucose and PEG-200 in distilled water, the clear solution was heated in a microwave oven for different times at $400 \mathrm{~W}$ and $2450 \mathrm{~Hz}$. At the end, the produced dark suspension of CNPs was used for desulfurization experiments without any further processing. In order to take transmission electron microscope (TEM) images of the solid phase of adsorbent, suspended CNPs were separated from solution as follows: a saturated solution of $\mathrm{NaCl}$ was added, and after agglomeration of the CNPs, the solid phase was separated from the liquid phase by centrifugation. After drying the sample at $110^{\circ} \mathrm{C}$ overnight, it was washed three times with distilled water in order to remove the $\mathrm{NaCl}$ and was dried again at the same condition.

\subsection{Design of experiment}

Variables optimization for the synthesis of CNPs was performed using a CCD, a standard RSM design. This approach limits the number of actual experiments performed while considering possible interaction between factors as well as individual variables and was suitable for fitting a quadratic surface (Asenjo et al. 2011; Kalavathy et al. 2009; Tan et al. 2008a). The selected variables in the present study are irradiation time $\left(X_{1}\right)$, water volume $\left(X_{2}\right)$, glucose weight $\left(X_{3}\right)$, and volume of polyethylene glycol $\left(X_{4}\right)$. The range and coded levels employed for the four factors in three levels, according to CCD design, are categorized in Table 1. Twenty-eight experimental runs, consisting of 18 cube points (for a full factorial), 8 star or axial points, and 4 center points, were generated by the principle of CCD (listed in Table 2) (Kalavathy et al. 2009). The order of these experiments was randomized in order to minimize the effect of uncertain variability in the monitored responses due to irrelevant factors (Asenjo et al. 2011). The quadratic polynomial model (Eq. 1) was chosen for fitting the experimental data:

$Y=b_{0}+\sum_{i=1}^{n} b_{i} X_{i}+\left(\sum_{i=1}^{n} b_{i i} X_{i}\right)^{2}+\sum_{i=1}^{n-1} \sum_{j=i=1}^{n} b_{i j} X_{i} X_{j}$,

where $Y$ is the response variable (i.e., removal efficiency (\%) of DBT), $b_{0}, b_{i}, b_{i i}$, and $b_{i j}$ represent the intercept, the linear, quadratic, and interaction coefficients, respectively.
Table 1 Experimental range and levels of the independent variables used for CCD

\begin{tabular}{llllll}
\hline Variable name & \multicolumn{2}{l}{ Range and coded factor levels } & \\
\cline { 2 - 6 } & -2 (low) & -1 & 0 & +1 & +2 (high) \\
\hline Irradiation time $(t), X_{1}, \mathrm{~min}$ & 7 & 9 & 11 & 13 & 15 \\
Water volume $(\mathrm{W}), X_{2}, \mathrm{~mL}$ & 7 & 13 & 19 & 25 & 31 \\
Glucose weight $(\mathrm{G}), X_{3}, \mathrm{~g}$ & 0.2 & 1.4 & 2.6 & 3.8 & 5 \\
Polyethylene glycol volume $(\mathrm{PEG}), X_{4}, \mathrm{~mL}$ & 8 & 13 & 18 & 23 & 28 \\
\hline
\end{tabular}


Table 2 List of experiments according to the CCD

\begin{tabular}{|c|c|c|c|c|c|}
\hline \multirow[t]{2}{*}{ Experiment number } & \multicolumn{4}{|c|}{ Factors levels } & \multirow[t]{2}{*}{ Response: removal, \% } \\
\hline & $t, \min$ & $\mathrm{W}, \mathrm{mL}$ & $\mathrm{G}, \mathrm{g}$ & $\mathrm{PEG}, \mathrm{mL}$ & \\
\hline 1 & 9 & 13 & 1.4 & 13 & 77.9 \\
\hline 2 & 13 & 13 & 1.4 & 13 & 78.3 \\
\hline 3 & 9 & 25 & 1.4 & 13 & 76.1 \\
\hline 4 & 13 & 25 & 1.4 & 13 & 77.8 \\
\hline 5 & 9 & 13 & 3.8 & 13 & 71.1 \\
\hline 6 & 13 & 13 & 3.8 & 13 & 75.3 \\
\hline 7 & 9 & 25 & 3.8 & 13 & 69.2 \\
\hline 8 & 13 & 25 & 3.8 & 13 & 73.9 \\
\hline 9 & 9 & 13 & 1.4 & 23 & 80.1 \\
\hline 10 & 13 & 13 & 1.4 & 23 & 81.2 \\
\hline 11 & 9 & 25 & 1.4 & 23 & 79.5 \\
\hline 12 & 13 & 25 & 1.4 & 23 & 80.6 \\
\hline 13 & 9 & 13 & 3.8 & 23 & 76.4 \\
\hline 14 & 13 & 13 & 3.8 & 23 & 77.9 \\
\hline 15 & 9 & 25 & 3.8 & 23 & 76.4 \\
\hline 16 & 13 & 25 & 3.8 & 23 & 78.7 \\
\hline 17 & 7 & 19 & 2.6 & 18 & 71.2 \\
\hline 18 & 15 & 19 & 2.6 & 18 & 78.9 \\
\hline 19 & 11 & 7 & 2.6 & 18 & 79.5 \\
\hline 20 & 11 & 31 & 2.6 & 18 & 76.9 \\
\hline 21 & 11 & 19 & 0.2 & 18 & 80.1 \\
\hline 22 & 11 & 19 & 5 & 18 & 75.4 \\
\hline 23 & 11 & 19 & 2.6 & 8 & 71.7 \\
\hline 24 & 11 & 19 & 2.6 & 28 & 78.7 \\
\hline $25^{\mathrm{a}}$ & 11 & 19 & 2.6 & 18 & 77.8 \\
\hline $26^{\mathrm{a}}$ & 11 & 19 & 2.6 & 18 & 77.3 \\
\hline $27^{\mathrm{a}}$ & 11 & 19 & 2.6 & 18 & 78.0 \\
\hline $28^{\mathrm{a}}$ & 11 & 19 & 2.6 & 18 & 77.6 \\
\hline
\end{tabular}

${ }^{a}$ Center points

A statistical package, 'Design Expert' software (Version 7.1.3, Stat-Ease Inc., Minneapolis, USA), was used to analyze and calculate the polynomial coefficients of the quadratic equation and to evaluate the statistical significance of the equation.

\subsection{Batch adsorption measurements}

In all experiments, a glass bottle containing CNPs and model fuel, prepared by dissolving sulfur-containing compounds in heptane with an initial concentration of 500 ppmw, was placed in a stirred thermostated batch system at $25.0 \pm 0.1{ }^{\circ} \mathrm{C}$. At first, desulfurization performance of sorbents (synthesized under different conditions) was evaluated in order to determine the optimum synthesis situation of CNPs using RSM. For this purpose, $2.5 \mathrm{~g}$ of model fuel containing DBT 500 ppmw, as a representative sulfur compound because of having a typical structure, was added to $5 \mathrm{~g}$ of CNPs solution. After $5 \mathrm{~min}$ of high-speed magnetic stirring, a sample was taken from the organic phase to evaluate the residual DBT content and estimate the removal efficiency. Afterward, the best adsorbent synthesized under optimum conditions was chosen for further investigations. The procedure used for kinetic tests was as follows: $10 \mathrm{~g}$ of CNPs suspension and $5 \mathrm{~g}$ of model fuel were mixed well under high-speed magnetic stirring, and then the sampling was done at desired time intervals. To study the effect of the mass of CNPs on adsorbent performance, $2.5 \mathrm{~g}$ of the DBT containing solutions, with an initial concentration of 1000 ppmw, was added to bottles containing different amounts of CNPs, from 0.1 to $8 \mathrm{~g}$, and they were mixed well under stirring and left to 
equilibrate for $20 \mathrm{~min}$. Afterward, the removal efficiency of adsorbent was estimated by $\% R=\left(C_{i}-C_{f}\right) \times 100 / C_{i}$, where $C_{i}$ and $C_{f}$ are the initial and final concentrations of sulfur compound (ppmw), respectively, and $\% \mathrm{R}$ is the removal efficiency. These experiments were repeated for other sulfur compounds (i.e., BT and DMDBT). In all the tests, the sulfur compound was dissolved in heptane, and then the initial and residual concentration of it was determined using UV-Visible spectrophotometry at the related $\lambda_{\max }$ (Fallah and Azizian 2012b).

\subsection{Characterization methods}

Carbon nanoparticles synthesized under the optimum condition were characterized by using a transmission electron microscope (Philips EM208), photoluminescence (PL) (LS50 spectrofluorimeter, Perkin Elmer), Fourier transform infrared spectroscope (FTIR) (Perkin Elmer), and UV-Visible spectrophotometer (Spekol 2000 spectrophotometer, Analytik Jena).

\section{Results and discussion}

\subsection{Development of regression model equation}

The experimental conditions, including value of each parameter, are presented in Table 2 along with the observed response. Analysis of variance (ANOVA) for the response surface quadratic model is summarized in Table 3. The suitability of the models was justified through ANOVA. From the ANOVA, the model $F$ value of 18.54 implied that the model was statistically significant. Values of 'Prob $>F$ ' less than 0.0500 indicated that the model terms were significant and those greater than 0.1000 were not. In this case, $X_{1}, X_{2}, X_{3}, X_{4}, X_{1} X_{3}, X_{3} X_{4}, X_{1}^{2}$ and $X_{4}^{2}$ were significant. Therefore, the final regression equation for removal efficiency in terms of actual factors after ignoring the insignificant terms is shown in Eq. (2):

$$
\begin{aligned}
R= & 51.72+3.70 X_{1}-0.08 X_{2}-5.45 X_{3}+1.03 X_{4} \\
& +0.22 X_{1} X_{3}+0.09 X_{3} X_{4}-0.16 X_{1}^{2}-0.02 X_{4}^{2}
\end{aligned} .
$$

A positive sign in front of the terms indicated a synergistic effect, whereas a negative sign showed antagonistic results. The quality of the model equation was further estimated using the correlation coefficients $\left(r^{2}\right)$ (Tan et al. 2008b). $r^{2}$ of this model with the value of 0.934 demonstrated a good agreement between experimental data and the model prediction. Figure 1 shows the predicted versus actual (experimental) removal efficiency. As mentioned above and represented in Fig. 1, this model was adequate to predict response.

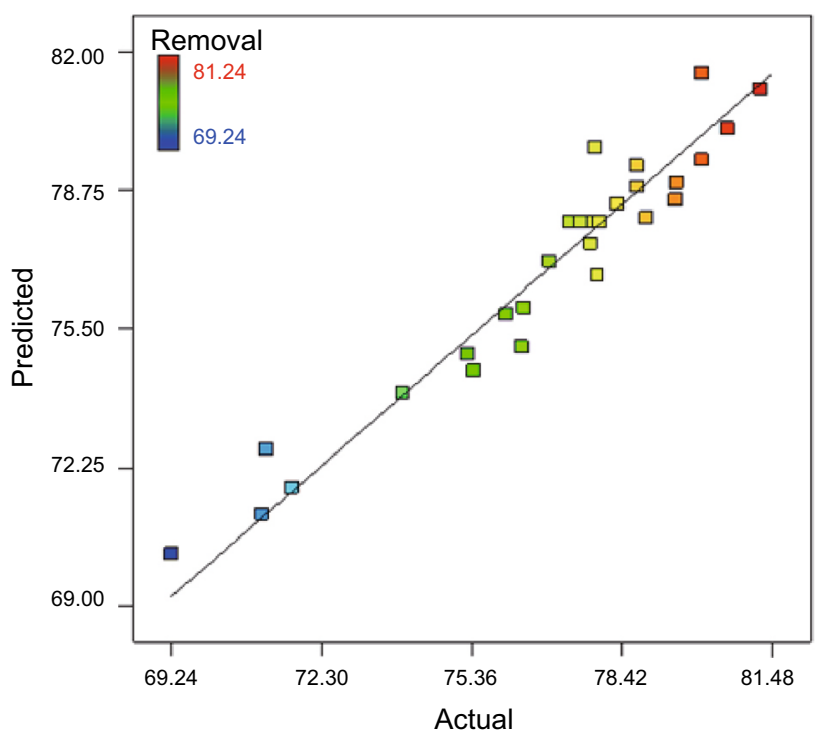

Fig. 1 Comparison plot between the experiments and the model predicted for DBT removal efficiency

\subsection{Optimization of CNPs synthesis for the removal of DBT}

According to Table 3, the volume of polyethylene glycol (PEG) $\left(X_{4}\right)$, weight of glucose $(\mathrm{G})\left(X_{3}\right)$, and irradiation time $(t)\left(X_{1}\right)$ showed the large $F$ value of $92.40,79.11$, and 47.79 , respectively, indicating that these factors had more significant effects on adsorbent performance in removing of DBT. From Eq. (2), $t$ and PEG were found to have synergistic effects on the removal efficiency of DBT in contrast with the effect of $\mathrm{G}$ and water volume $(W)$. Besides, the quadratic effect of irradiation time $\left(X_{1}^{2}\right)$ and PEG volume $\left(X_{4}^{2}\right)$ on the DBT uptake was relatively significant with antagonist effects. So, it was expected that an increase in irradiation time could increase efficiency and then decrease it after reaching a maximum value. This is very clear from the one factor plot of varying responses with changing irradiation time (the other variables were constant at central levels) (Fig. 2). Similar behavior was observed for PEG volume. Equation (2) suggests that the weight of glucose variable interacted with the time of irradiation and volume of PEG. But these interactions $\left(\left(X_{1}\right.\right.$ $\left.X_{3}\right)$ and $\left.\left(X_{3} X_{4}\right)\right)$ and also the volume of water $\left(X_{2}\right)$ have less significant effects as shown by the low $F$ value. Isoresponse counter plots (three dimensional response surface contour plots), displayed in Fig. 3a-c, are the graphical representations of the regression equation. The main object of the response surface was finding the optimum values of the factors, so the response variable was maximized. Each contour curve represents a combination of irradiation time $(t)$ and polyethylene glycol (PEG) with the volume of water (W) and weight of glucose 
Table 3 Analysis of variance (ANOVA) for response surface quadratic model

\begin{tabular}{lcccrr}
\hline Source & Sum of square & Degree of freedom & Mean square & F value & probe $>$ F \\
\hline Model & 239.36 & 14 & 17.10 & 18.54 & $<0.0001$ \\
$X_{1}$ & 44.06 & 1 & 44.06 & 47.79 & $<0.0001$ \\
$X_{2}$ & 5.15 & 1 & 5.15 & 5.59 & 0.0343 \\
$X_{3}$ & 72.94 & 1 & 72.94 & 79.11 & $<0.0001$ \\
$X_{4}$ & 85.20 & 1 & 85.20 & 92.40 & $<0.0001$ \\
$X_{1} X_{2}$ & 0.42 & 1 & 0.42 & 0.46 & 0.5103 \\
$X_{1} X_{3}$ & 4.35 & 1 & 4.35 & 4.71 & 0.0490 \\
$X_{1} X_{4}$ & 1.55 & 1 & 1.55 & 1.68 & 0.2173 \\
$X_{2} X_{3}$ & 0.10 & 1 & 0.10 & 0.11 & 0.7443 \\
$X_{2} X_{4}$ & 1.66 & 1 & 1.66 & 1.80 & 0.2021 \\
$X_{3} X_{4}$ & 4.60 & 1 & 4.60 & 4.99 & 0.0437 \\
$X_{1}^{2}$ & 8.56 & 1 & 8.56 & 9.28 & 0.0094 \\
$X_{2}^{2}$ & 0.93 & 1 & 0.93 & 1.06 & 0.3223 \\
$X_{3}^{2}$ & 0.13 & 1 & 0.13 & 0.14 & 0.7159 \\
$X_{4}^{2}$ & 7.48 & 1 & 7.48 & 8.11 & 0.0137 \\
\hline
\end{tabular}

Significant at "probe $>$ F" less than 0.05

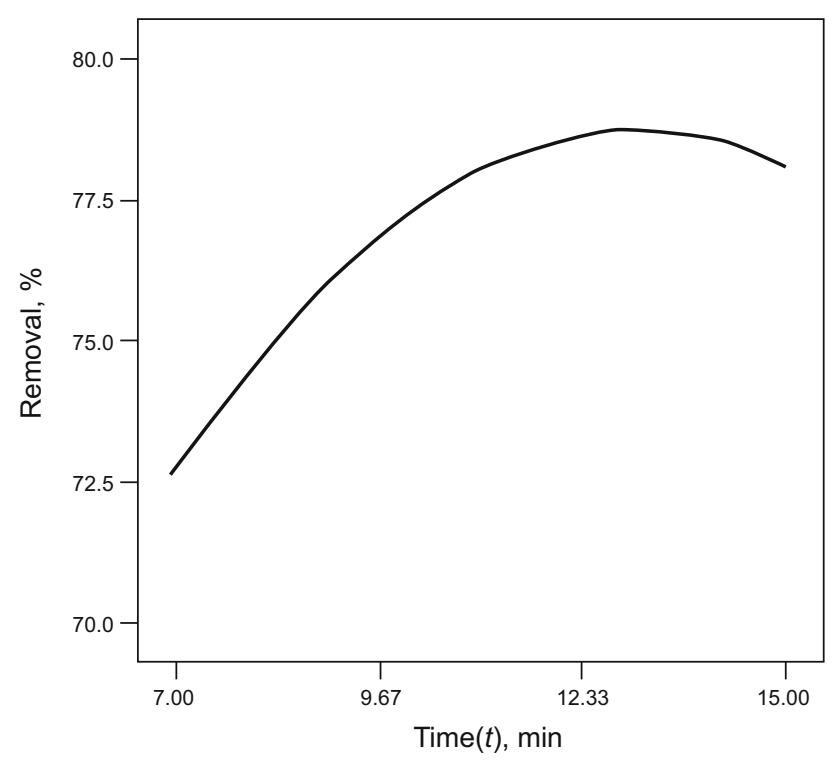

Fig. 2 One factor plot showing the effect of irradiation time on removal efficiency of DBT (the other factors were maintained at their central points)

(G) maintained at different levels. The predicted maximum response was indicated by the surface confined in the smallest ellipse in the contour diagram. Elliptical contours are obtained when there is a perfect interaction between the independent variables (Tanyildizi et al. 2005). As can be seen from Fig. 3a-c, the removal percentage of DBT increased with the increase in $t$ and PEG but decreased with the increase of $\mathrm{W}$ and $\mathrm{G}$. The highest removal of DBT was obtained when both latter variables (W and $\mathrm{G}$ ) were at the minimum point within the range studied and the response surface turned into an ellipse in the last figure (Fig. 3c).
Therefore, the optimal experimental conditions for obtaining optimized dispersed CNPs were found to be $t=$ $11 \mathrm{~min}, \mathrm{PEG}=20 \mathrm{~mL}, G=0.2 \mathrm{~g}$ and $W=7 \mathrm{~mL}$. The predicted maximum removal efficiency of DBT by CNPs was $83 \%$.

\subsection{Characterization of the prepared CNPs at optimum conditions}

The optimum conditions for preparation of CNPs with maximum removal efficiency of DBT from fuel were found in the above section. The prepared CNPs were characterized by different methods, which will be explained below. Although the photoluminescence (PL) spectra for CNPs in Fig. 4 are similar to those of the reported data (Fallah and Azizian 2012a), but the UV-Visible spectrum (Fig. 5) of CNPs synthesized in optimum conditions is different from the reports in literature (Fallah and Azizian 2012a; Fallah et al. 2012). In this work, maximum absorption was observed at a wavelength of $220 \mathrm{~nm}$ (Fig. 5), while in the previous reports, $\lambda_{\max }$ was at $280 \mathrm{~nm}$. According to the report by Tian et al. (2010), the UV-Visible spectra of carbon nanoparticles contain a major absorption peak at around $200 \mathrm{~nm}$ (peak A) and another broad peak at approximately $300 \mathrm{~nm}$ (peak B). The first absorption peak $\mathrm{A}$ is attributed to the $\pi-\pi^{*}$ electronic transitions of internal $\left(\mathrm{sp}^{2}\right)$ graphitic carbons, whereas the second absorption peak B is most likely related to $n-\pi^{*}$ electron transfer of carbonyl $(\mathrm{C}=\mathrm{O})$ groups (e.g., aldehydes and ketones) on the CNPs surface. Upon heating, the peak A remained, whereas the peak B diminished (Tian et al. 2010). The temperatures of CNPs solutions (synthesized in the present (CNPs II) and previous (CNPs I) (Fallah et al. 2012) were 


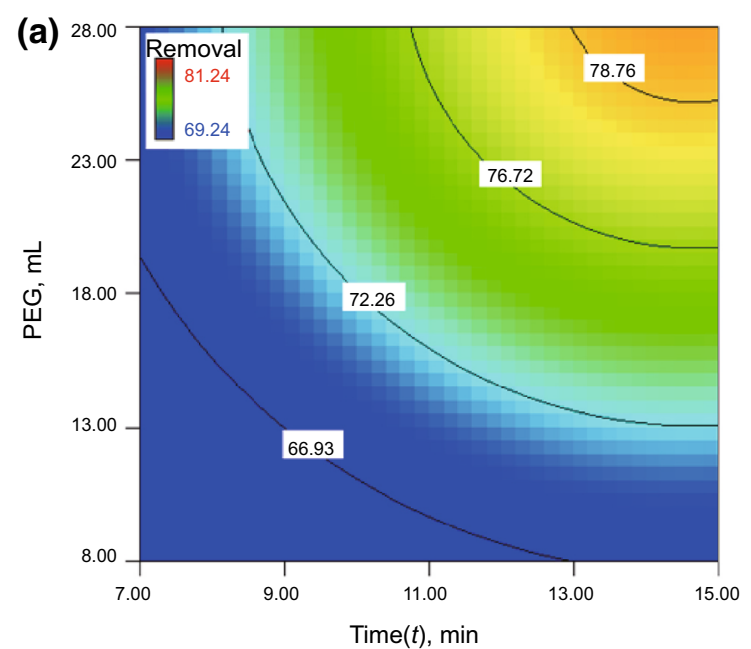

(b)

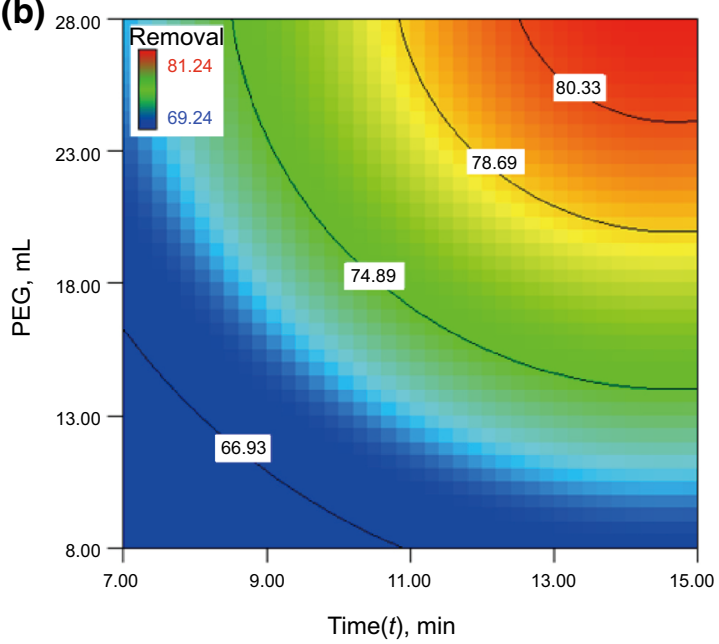

(c)

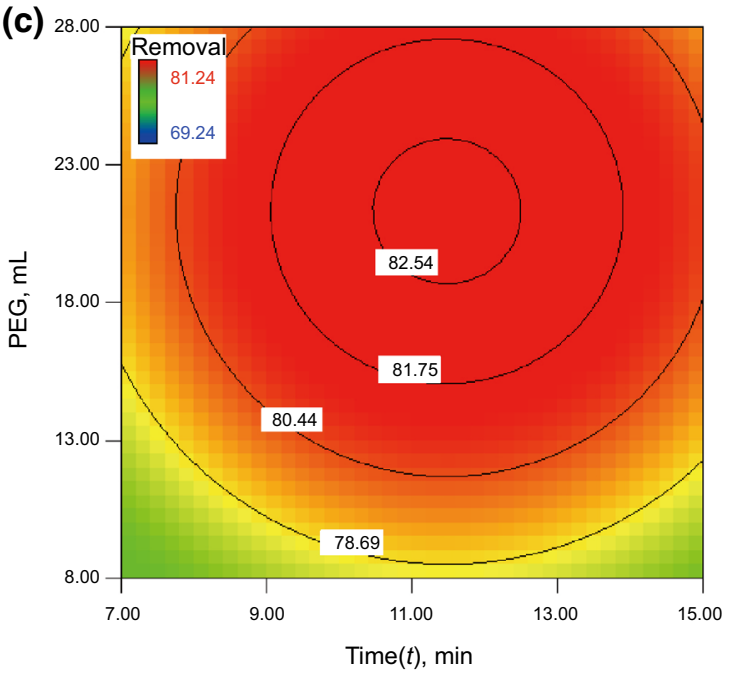

Fig. 3 Isoresponse counter plot showing the effect of PEG volume, irradiation time, and their mutual effect on removal efficiency of DBT by CNPs at different levels of water volume (W) and glucose weight (G) (a W and G at high levels, b W at low level and G at high level, c $\mathrm{W}$ and $\mathrm{G}$ at low levels)

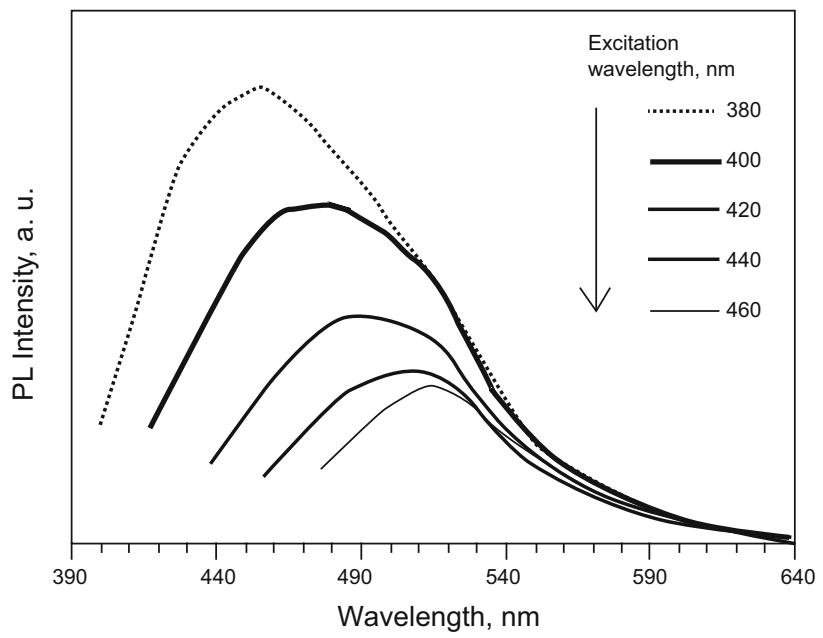

Fig. 4 Photoluminescence (PL) spectra of the prepared CNPs with different excitation wavelengths

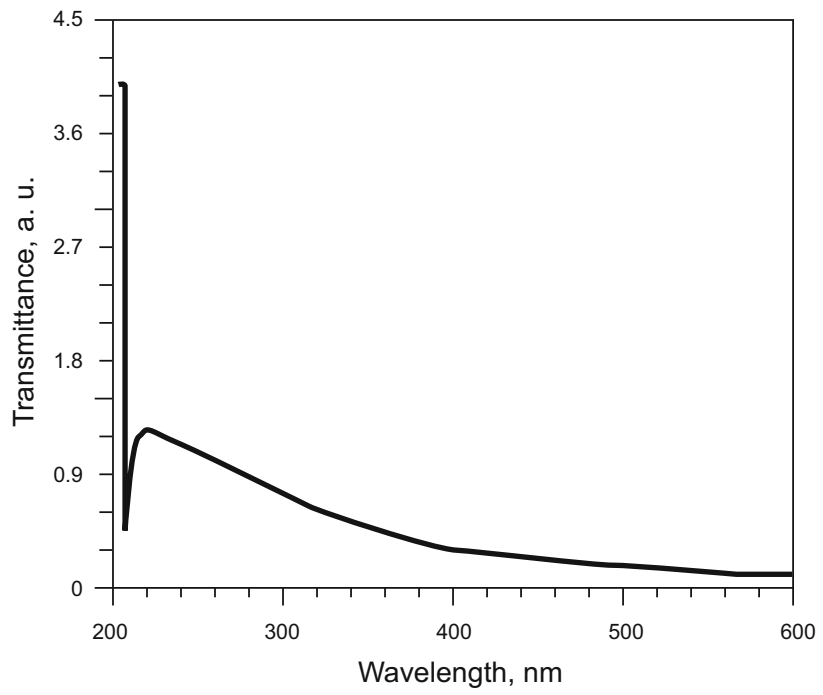

Fig. 5 UV/Visible spectra of the prepared CNPs in suspension

measured to be $265{ }^{\circ} \mathrm{C}$ and $240{ }^{\circ} \mathrm{C}$, respectively, using a thermocouple immediately after bringing the samples out of the microwave. The increase in temperature may cause the surface functional groups containing oxygen to be decomposed. In fact, the shift in the UV-Visible spectrum of CNPs from $280 \mathrm{~nm}$ (Fallah et al. 2012) to $220 \mathrm{~nm}$ in the present work suggests the removal of carbonyl groups from the nanoparticles surface. The above interpretation was further justified by the result from FTIR measurements (Fig. 6). It can be seen that the overall FTIR spectrum of solid CNPs (Fig. 6a) prepared in this work was similar to that of the carbon nanoparticles prepared in the previous study (Fig. 6b) (Fallah et al. 2012), and only some differences in the intensity ratio of a number of peaks were 

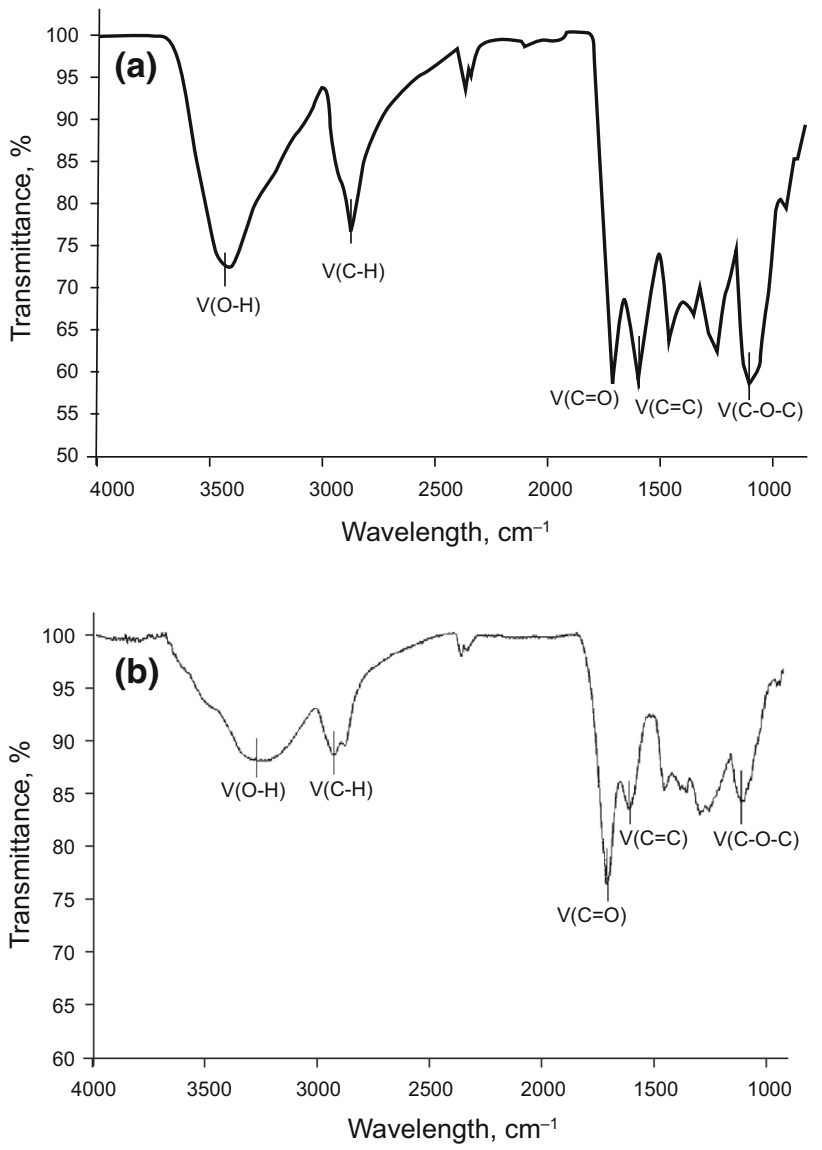

Fig. 6 FTIR spectra of the solid CNPs prepared in a the present study and b previous study (Fallah et al. 2012)

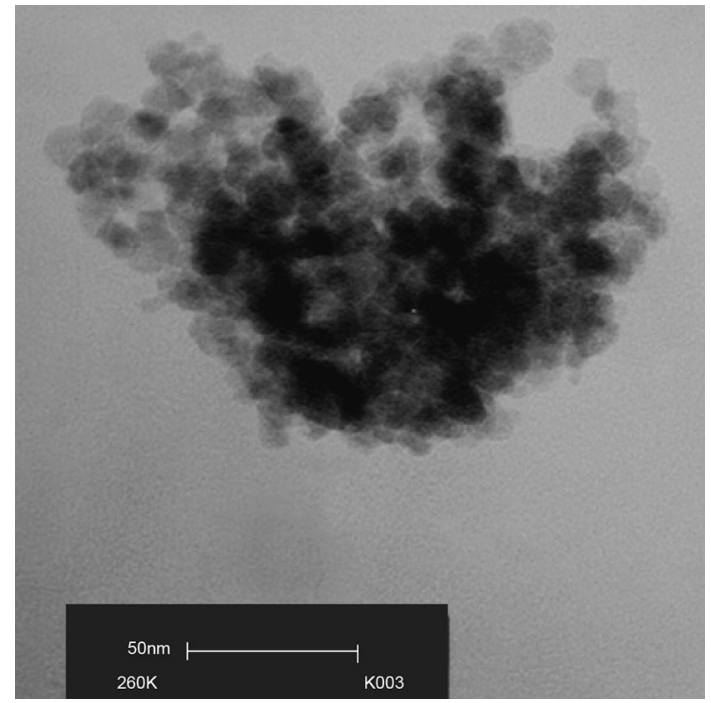

Fig. 7 TEM image of the solid CNPs synthesized under optimal conditions in this work

observed. The ratio of the $\mathrm{C}=\mathrm{O}$ to $\mathrm{C}=\mathrm{C}$ absorbance peak decreased for CNPs II in comparison to CNPs I. This observation is in agreement with the above discussions.
TEM images were obtained for further characterization of CNPs prepared under the optimum conditions. As can be seen from Fig. 7, CNPs are spherical less than $10 \mathrm{~nm}$ in size, smaller than the previous reports (Fallah and Azizian 2012a; Fallah et al. 2012).

\subsection{Kinetics of desulfurization by the prepared CNPs}

Figure 8 shows the effect of agitation time on removal of BT, DBT, and DMDBT by the synthesized CNPs. It can be seen that the concentrations of BT, DBT, and DMDBT decreased to 126,86 , and 241 ppmw and equilibrium was achieved within $90 \mathrm{~s}$ for BT and DBT and $120 \mathrm{~s}$ for DMDBT with removal efficiency values of $75 \%, 83 \%$ and $52 \%$, respectively. The rate of desulfurization by the prepared CNPs is much higher than the previously reported data for CNPs where the system approached equilibrium after 15 min (Fallah and Azizian 2012a). This observation was probably due to the size of CNPs synthesized in different conditions (synthesis conditions of this work: $t=$ $11 \mathrm{~min}, \mathrm{PEG}=20 \mathrm{~mL}, G=0.2 \mathrm{~g}$ and $W=7 \mathrm{~mL}$ and previously reported work (Fallah and Azizian 2012a): $t=$ $9 \mathrm{~min}, \mathrm{PEG}=10 \mathrm{~mL}, G=2 \mathrm{~g}$ and $W=10 \mathrm{~mL}$ ). The smaller particles lead to an increase in the surface area of adsorbent, and therefore, the uptake rate of adsorbate on the sorbent has been increased as a consequence of increasing the surface area of adsorbent. As noted in Sect. 3.3, the present CNPs of less than $10 \mathrm{~nm}$ are finer than previously reported CNPs (Fallah and Azizian 2012a; Fallah et al. 2012). The experimental kinetic data of sulfur compounds' uptake on CNPs were best fitted using a firstorder kinetic model, $\ln (1-F)=-k t$, where

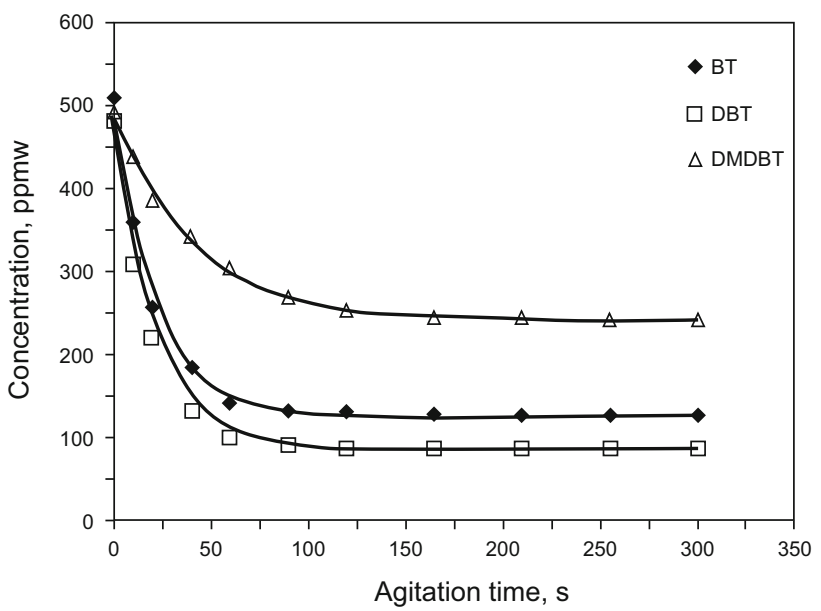

Fig. 8 Concentration variation of different sulfur compounds with time using CNPs. Symbols are the experimental values and lines are the predicted values from the first-order kinetic model. Experimental conditions: mass ratio of $\mathrm{CNP} /$ model oil $=1: 2$, sulfur compound concentration $500 \mathrm{ppmw}, T=25^{\circ} \mathrm{C}$ 


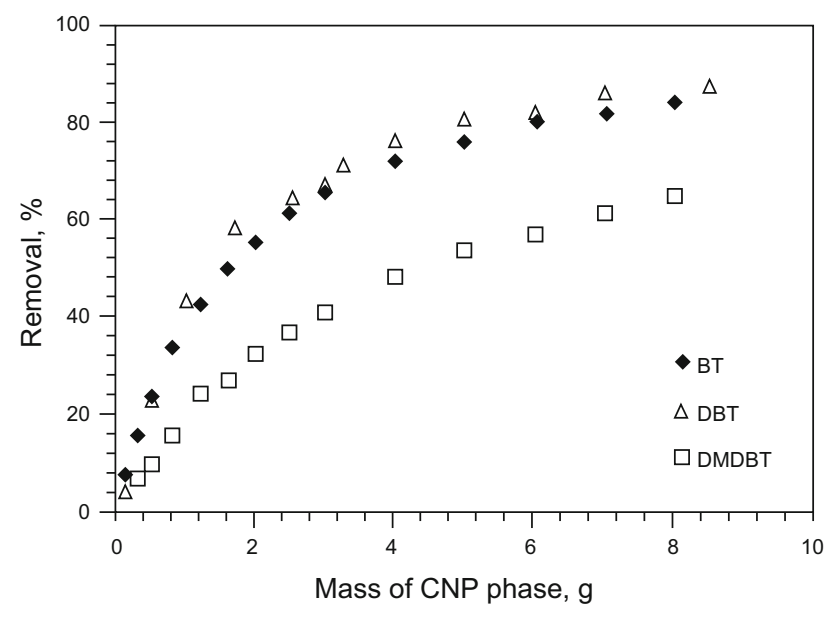

Fig. 9 Effect of CNP mass on BT, DBT, and DMDBT removal. Sulfur compound concentration $1000 \mathrm{ppmw}, T=25^{\circ} \mathrm{C}$

$F=\left(C_{0}-C_{t}\right) /\left(C_{0}-C_{e}\right) ; C_{0}, C_{e}$, and $C_{t}$ are concentrations at initial, equilibrium, and at any time, respectively. Solid lines in Fig. 8 represent the predicted values by this model. The obtained rate constants $(k)$ for BT, DBT, and DMDBT at $25{ }^{\circ} \mathrm{C}$ are $0.046,0.045$, and $0.025 \mathrm{~s}^{-1}$, respectively, which confirm the higher rate of BT and DBT removal.

\subsection{Effect of the mass of CNPs}

The effect of the mass of CNPs on the removal performance was investigated, and the results are presented in Fig. 9. It is clear that removal efficiency values of BT, DBT, and DMDBT were increased rapidly with increasing the mass of CNPs up to $5 \mathrm{~g}$ and then increased very slowly.

\section{Conclusion}

A central composite design (CCD) was conducted to optimize the process conditions for the synthesis of CNPs in order to obtain an adsorbent with higher removal efficiency for sulfur compounds. The optimum conditions for preparation of CNPs with the highest rate of desulfurization was $t=$ $11 \mathrm{~min}, \mathrm{PEG}=20 \mathrm{~mL}, G=0.2 \mathrm{~g}$, and $W=7 \mathrm{~mL}$. Kinetic studies revealed that the rate of the sulfur removal process was very rapid and equilibrium was achieved within about 2 min. The experimental kinetic data were best fitted with a first-order kinetic model. The rate of desulfurization of liquid fuel by the prepared CNPs in the present work was seven times higher than that of the previously reported CNPs. Therefore, the prepared CNPs, which was synthesized by a simple and environmentally friendly method and which can be removed simply from the operation medium because of immiscibility with organic phase (fuel), is one of the best sorbents for the desulfurization of fuel.
Acknowledgments The financial support from Bu-Ali Sina University was gratefully acknowledged.

Open Access This article is distributed under the terms of the Creative Commons Attribution 4.0 International License (http://creative commons.org/licenses/by/4.0/), which permits unrestricted use, distribution, and reproduction in any medium, provided you give appropriate credit to the original author(s) and the source, provide a link to the Creative Commons license, and indicate if changes were made.

\section{References}

Asenjo NG, Botas C, Blanco C, et al. Synthesis of activated carbons by chemical activation of new anthracene oil-based pitches and their optimization by response surface methodology. Fuel Process Technol. 2011;92(10):1987-92.

Bezerra MA, Santelli RE, Oliveira EP, et al. Response surface methodology (RSM) as a tool for optimization in analytical chemistry. Talanta. 2008;76(5):965-77.

Blanco-Brieva G, Campos-Martin JM, Al-Zahrani SM, et al. Effectiveness of metal-organic frameworks for removal of refractory organo-sulfur compound present in liquid fuels. Fuel. 2011;90(1):190-7.

Bösmann A, Datsevich L, Jess A, et al. Deep desulfurization of diesel fuel by extraction with ionic liquids. Chem Commun. 2001;23:2494-5.

Fallah RN, Azizian S. Rapid and facile desulfurization of liquid fuel by carbon nanoparticles dispersed in aqueous phase. Fuel. 2012a;95:93-6.

Fallah RN, Azizian S. Removal of thiophenic compounds from liquid fuel by different modified activated carbon cloths. Fuel Process Technol. 2012b;93(1):45-52.

Fallah RN, Azizian S, Reggers G, et al. Effect of aromatics on the adsorption of thiophenic sulfur compounds from model diesel fuel by activated carbon cloth. Fuel Process Technol. 2014;119:278-85.

Fallah RN, Azizian S, Reggers G, et al. Selective desulfurization of model diesel fuel by carbon nanoparticles as adsorbent. Ind Eng Chem Res. 2012;51(44):14419-27.

Farag H, Whitehurst DD, Mochida I. Synthesis of active hydrodesulfurization carbon-supported Co-Mo catalysts. Relationships between preparation methods and activity/selectivity. Ind Eng Chem Res. 1998;37(9):3533-9.

Hernandez SP, Fino D, Russo N. High performance sorbents for diesel oil desulfurization. Chem Eng Sci. 2010;65(1):603-9.

Jiang Z, Liu Y, Sun X, et al. Activated carbons chemically modified by concentrated $\mathrm{H}_{2} \mathrm{SO}_{4}$ for the adsorption of the pollutants from wastewater and the dibenzothiophene from fuel oils. Langmuir. 2003;19(3):731-6.

Kalavathy MH, Regupathi I, Pillai MG, et al. Modelling, Analysis and optimization of adsorption parameters for $\mathrm{H}_{3} \mathrm{PO}_{4}$ activated rubber wood sawdust using response surface methodology (RSM). Colloids Surf, B. 2009;70(1):35-45.

Lara G, Escobar J, De Los Reyes JA, et al. Dibenzothiophene HDS over sulphided CoMo on high-silica USY zeolites. Can J Chem Eng. 2005;83(4):685-94.

Liu B, Zhu Y, Liu S, Mao J. Adsorption equilibrium of thiophenic sulfur compounds on the $\mathrm{Cu}-\mathrm{BTC}$ metal-organic framework. J Chem Eng Data. 2012;57(4):1326-30.

Puri S, Beg QK, Gupta R. Optimization of alkaline protease production from bacillus sp. by response surface methodology. Curr Microbiol. 2002;44(4):286-90. 
Shi F, Hammoud M, Thompson LT. Selective adsorption of dibenzothiophene by functionalized metal organic framework sorbents. Appl Catal B. 2011;103(3-4):261-5.

Song CS, Ma XL. New design approaches to ultra-clean diesel fuels by deep desulfurization and deep dearomatization. Appl Catal B. 2003;41(1-2):207-38.

Song H, Wan X, Sun X. Preparation of Agy zeolites using microwave irradiation and study on their adsorptive desulphurisation performance. Can J Chem Eng. 2013;91(5):915-23.

Tan IAW, Ahmad AL, Hameed BH. Optimization of preparation conditions for activated carbons from coconut husk using response surface methodology. Chem Eng J. 2008a;137(3): 462-70.

Tan IAW, Ahmad AL, Hameed BH. Preparation of activated carbon from coconut husk: optimization study on removal of 2,4,6trichlorophenol using response surface methodology. J Hazard Mater. 2008b;153(1-2):709-17.
Tanyildizi MS, Ozer D, Elibol M. Optimization of $\alpha$-amylase production by Bacillus sp. using response surface methodology. Process Biochem. 2005;40(7):2291-6.

Tian L, Song Y, Changa X, et al. Hydrothermally enhanced photoluminescence of carbon nanoparticles. ScriptaMaterialia. 2010;62(11):883-6.

Yang Y, Lu H, Ying P, et al. Selective dibenzothiophene adsorption on modified activated carbons. Carbon. 2007;45(15):3042-4.

Zhang S, Zhang Q, Zhang ZC. Extractive desulfurization and denitrogenation of fuels using ionic liquids. Ind Eng Chem Res. 2004;43(2):614-22.

Zhu H, Wang X, Li Y, et al. Microwave synthesis of fluorescent carbon nanoparticles with electrochemiluminescence properties. Chem Commun. 2009;34:5118-20. 